\title{
AN INEQUALITY FOR EXPECTED VALUES OF SAMPLE QUANTILES ${ }^{1}$
}

\author{
By W. R. VAN ZWET \\ University of Leiden
}

1. Introduction. Let $F$ be a continuous distribution function on $R^{1}$ that is strictly increasing on the (finite or infinite) open interval $I$ where $0<F<1$, and let $G$ denote the inverse of $F$. For $n=1,2, \cdots$ and $0<\lambda<1$, let

$$
\begin{array}{r}
\gamma_{n}(\lambda)=[\Gamma(n+1) / \Gamma(\lambda(n+1)) \Gamma((1-\lambda)(n+1))] \int_{0}^{1} G(y) y^{\lambda(n+1)-1} \\
\cdot(1-y)^{(1-\lambda)(n+1)-1} d y .
\end{array}
$$

Obviously, if $X_{i: n}$ denotes the $i$ th order statistic of a sample of size $n$ from the parent distribution $F$, then

$$
\gamma_{n}(i /(n+1))=E X_{i: n}, \quad i=1,2, \cdots, n .
$$

We shall call $\gamma_{n}(\lambda)$ the expected value of the $\lambda$-quantile of a sample of size $n$ from $F$, even though this interpretation is meaningless when $\lambda(n+1)$ is not an integer. We shall assume that for some $\lambda$ the integral converges for sufficiently large $n$, which ensures that the same will hold for every $0<\lambda<1$. By making minor changes in W. Hoeffding's proof in [2], one shows that $\gamma_{n}$ converges to $G$ on $(0,1)$ for $n \rightarrow \infty$.

Consider another continuous distribution function $F^{*}$ that is strictly increasing on the interval $I^{*}$ where $0<F^{*}<1$, and let $G^{*}, \gamma_{n}{ }^{*}$ and $X_{i: n}^{*}$ be defined for $F^{*}$ analogous to $G, \gamma_{n}$ and $X_{i: n}$ for $F$. Furthermore let

$$
\phi(x)=G^{*} F(x),
$$

In [5] the author studied the following order relations between $F$ and $F^{*}$ :

$$
\begin{aligned}
& \phi \text { is convex on } I \text {; } \\
& F \text { and } F^{*} \text { represent symmetric distributions and } \phi \text { is concave- } \\
& \text { convex on } I \text {. }
\end{aligned}
$$

If $x_{0}$ denotes the median of $F$, relation (1.4) implies that $\phi$ is antisymmetric about $x_{0}$ (i.e. $\left.\phi\left(x_{0}+x\right)+\phi\left(x_{0}-x\right)=2 \phi\left(x_{0}\right)\right)$ and hence that $\phi$ is concave for $x<x_{0}$ and convex for $x>x_{0}$.

Let $\phi_{n}$ be the function that maps the expected value of the $\lambda$-quantiles of a sample of size $n$ from $F$ on the corresponding quantities for $F^{*}$ :

$$
\phi_{n}(x)=\gamma_{n}^{*} \gamma_{n}^{-1}(x) \text {. }
$$

Received 21 November 1966; revised 12 June 1967.

${ }^{1}$ Report S 369, Mathematisch Centrum, Amsterdam. 
For $n \rightarrow \infty, \phi_{n}$ will converge to the function $\phi$ on $I$ that maps the population quantiles of $F$ on those of $F^{*}$. It is shown in this note that if relations (1.3) or (1.4) hold, $\phi_{n}$ shares the convexity or concave-convexity of $\phi$, and the convergence of $\phi_{n}$ to $\phi$ is monotone. The convexity property yields a theorem on the behavior of the ratio of expected values of spacings of consecutive order statistics from $F$ and $F^{*}$. Simple applications are given in Section 3.

\section{The results.}

Theorem 2.1. If condition (1.3) holds, $\phi_{n}(x)$ is convex in $x$ for fixed $n$, and non-increasing in $n$ for fixed $x$.

Proof. For each fixed $n$ the densities

$$
\begin{aligned}
f_{\lambda}(y)=[\Gamma(n+1) / \Gamma(\lambda(n+1)) \Gamma((1-\lambda)(n+1))] \\
\cdot y^{\lambda(n+1)-1}(1-y)^{(1-\lambda)(n+1)-1}
\end{aligned}
$$

constitute a one-parameter exponential family for $0<\lambda, y<1$, and consequently the family is strictly totally positive of order $\infty$ in $\lambda$ and $y$ (cf. [3]). According to a slight elaboration of a result due to $\mathrm{S}$. Karlin that is given in [4], the convexity of $\phi_{n}$ follows from the definition of $\gamma_{n}$ and $\gamma_{n}{ }^{*}$, the total positivity of $f_{\lambda}(y)$, the monotonicity of $F$ and the convexity of $\phi$. Also

$$
\gamma_{n}(\lambda)=\lambda \gamma_{n+1}(\lambda+(1-\lambda) /(n+2))+(1-\lambda) \gamma_{n+1}(\lambda-\lambda /(n+2))
$$

and the same holds for $\gamma_{n}{ }^{*}$. This is easily verified by adding integrands in expression (1.1). Hence, because of the convexity of $\phi_{n+1}$,

$$
\begin{aligned}
& \phi_{n+1} \gamma_{n}(\lambda) \\
& =\phi_{n+1}\left(\lambda \gamma_{n+1}(\lambda+(1-\lambda) /(n+2))+(1-\lambda) \gamma_{n+1}(\lambda-\lambda /(n+2))\right) \\
& \leqq \lambda \phi_{n+1} \gamma_{n+1}(\lambda+(1-\lambda) /(n+2))+(1-\lambda) \phi_{n+1} \gamma_{n+1}(\lambda-\lambda /(n+2)) \\
& =\lambda \gamma_{n+1}^{*}(\lambda+(1-\lambda) /(n+2))+(1-\lambda) \gamma_{n+1}^{*}(\lambda-\lambda /(n+2)) \\
& =\gamma_{n}{ }^{*}(\lambda),
\end{aligned}
$$

or, replacing $\gamma_{n}(\lambda)$ by $x, \phi_{n+1}(x) \leqq \gamma_{n}{ }^{*} \gamma_{n}{ }^{-1}(x)=\phi_{n}(x)$.

In the same vein we have

TheOREM 2.2. If condition (1.4) holds, $\phi_{n}(x)$ is antisymmetric concave-convex about $x_{0}$ for fixed $n$, and non-increasing in $n$ for fixed $x>x_{0}$.

Proof. Obviously $\phi_{n}$ is antisymmetric about $x_{0}$. Since $\phi$ is concave-convex, $G^{*}$ is a concave-convex function of $G$ and hence

$$
h(y)=G^{*}(y)-a-b G(y)
$$

can have at most three changes of sign on $(0,1)$ for any $a$ and $b$. If it does change sign three times, the signs occur in the order $(-,+,-,+)$ for increasing values of the argument. It follows from the variation diminishing property of totally positive kernels (cf. [3]) that

$$
\gamma_{n}^{*}(\lambda)-a-b \gamma_{n}(\lambda)=\int_{0}^{1} h(y) f_{\lambda}(y) d y
$$


changes sign at most three times; if it does have three sign changes, the signs occur in the order $(-,+,-,+)$. Substituting $\gamma_{n}(\lambda)=x$ we find that $\phi_{n}(x)-$ $a-b x$ possesses the same property for any $a$ and $b$. A simple geometrical argument based on the antisymmetry of $\phi_{n}$ shows that this implies that $\phi_{n}$ is concaveconvex about $x_{0}$. Since for $\lambda>\frac{1}{2}$

$$
(\lambda+(1-\lambda) /(n+2))+(\lambda-\lambda /(n+2))>1,
$$

and hence by the antisymmetry of $\gamma_{n+1}$

$$
\gamma_{n+1}(\lambda+(1-\lambda) /(n+2))+\gamma_{n+1}(\lambda-\lambda /(n+2))>2 x_{0}
$$

the inequality of (2.3) remains valid now that $\phi_{n}$ is antisymmetric and concaveconvex instead of convex. This completes the proof.

We note that in the proofs of Theorems 2.1 and 2.2 we have only made use of the total positivity of $f_{\lambda}(y)$. Exploiting the fact that the total positivity is strict, one finds that the convexity (or concave-convexity) in $x$ as well as the monotonicity in $n$ of $\phi_{n}(x)$ are strict, unless $\phi$ is linear on $I$.

The quantities $\gamma_{n}(\lambda)$ for non-integer $\lambda(n+1)$ were introduced to facilitate the discussion of $\lambda$-quantiles for fixed $\lambda$ and varying $n$. However, in considering the convexity of $\phi_{n}$ for fixed $n$, we may as.well restrict ourselves to the case where $i=\lambda(n+1)$ is an integer. Theorem 2.1 then states that if condition (1.3) holds, i.e. if $G^{*}$ is a convex function of $G$, then $E X_{i: n}^{*}$ is a convex function of $E X_{i: n}$ for varying $i$ and fixed $n$, i.e.

$$
\left(E X_{i+1: n}^{*}-E X_{i: n}^{*}\right) /\left(E X_{i+1: n}-E X_{i: n}\right)
$$

is non-decreasing in $i$ for fixed $n$. We recall that the proof of this assertion rests solely on the fact that the family (2.1), which for $i=\lambda(n+1)$ becomes

$$
f_{i: n}(y)=[n ! /(i-1) !(n-i) !] y^{i-1}(1-y)^{n-i},
$$

is totally positive of order infinity in $i$ and $y$ for fixed $n$. However, the family (2.5) is also totally positive of order infinity in $n$ and $(1-y)$ for fixed $i$. One easily verifies that this implies that $E X_{i: n}^{*}$ is also a convex function of $E X_{i: n}$ for varying $n$ and fixed $i$. Since $E X_{i: n}$ is decreasing in $n$ for fixed $i$, it follows that

$$
\left(E X_{i: n}^{*}-E X_{i: n+1}^{*}\right) /\left(E X_{i: n}-E X_{i: n+1}\right)
$$

is non-increasing in $n$. Using formula (2.2) for $\lambda(n+1)=i$, i.e.

$$
E X_{i: n}=[i /(n+1)] E X_{i+1: n+1}+[(n+1-i) /(n+1)] E X_{i: n+1},
$$
and the corresponding expression for $E X_{i: n}^{*}$, we find

$$
\begin{aligned}
\left(E X_{i: n}^{*}-E X_{i: n+1}^{*}\right) /\left(E X_{i: n}-\right. & \left.E X_{i: n+1}\right) \\
& =\left(E X_{i+1: n+1}^{*}-E X_{i: n+1}^{*}\right) /\left(E X_{i+1: n+1}-E X_{i: n+1}\right),
\end{aligned}
$$

and hence (2.4) is non-increasing in $n$.

By considering the distribution functions $1-F^{*}(-x)$ and $1-F(-x)$ in- 
stead of $F$ and $F^{*}$ one easily shows that

$$
\left(E X_{n-i+1: n}^{*}-E X_{n-i: n}^{*}\right) /\left(E X_{n-i+1: n}-E X_{n-i: n}\right)
$$

is non-increasing in $i$ and non-decreasing in $n$. The former conclusion is of course equivalent to the monotonicity in $i$ of (2.4). We have proved

Theorem 2.3. If condition (1.3) holds, the quantities (2.4) are non-decreasing in $i$ and non-increasing in $n$, whereas (2.7) is non-decreasing in $n$.

We note that the last assertion of the theorem may also be proved directly by using the total positivity of (2.5) in $i$ and $y$ for fixed $(n-i)$ and applying (2.6).

It may be of interest to point out the similarity of Theorem 2.3 to inequalities that were recently obtained by R. E. Barlow and F. Proschan [1] for the case where $F(0)=F^{*}(0)=0$ and $\phi$ is starshaped (i.e. $\phi(x) / x$ non-decreasing on $I$ ). By total positivity arguments similar to those given above they show that $E X_{i: n}^{*} / E X_{i: n}$ is non-decreasing in $i$ and non-increasing in $n$, whereas $E X_{n-i: n}^{*} /$ $E X_{n-i: n}$ is non-decreasing in $n$.

3. Applications. Let $F$ be the uniform distribution function on $(0,1)$. Then

$$
\gamma_{n}(\lambda)=\lambda \text { for } 0<\lambda<1,
$$

$\phi=G^{*}$ and $\phi_{n}=\gamma_{n}{ }^{*}$. If $F^{*}$ is differentiable on $I^{*}$, it satisfies conditions (1.3) or (1.4) if its density $F^{* \prime}$ is non-increasing on $I^{*}$, or symmetric and unimodal respectively. Consequently we have:

The expected value of the $\lambda$-quantile of a sample of size $n$ from a distribution with non-increasing density is a non-increasing function of $n$; if the density is symmetric and unimodal the conclusion remains valid for $\lambda>\frac{1}{2}$. Moreover, if $F^{* \prime}$ is non-increasing, $(n+1)\left(E X_{i+1: n}^{*}-E X_{i: n}^{*}\right)$ is non-decreasing in $i$ and non-increasing in $n$, whereas $(n+1)\left(E X_{n-i+1: n}^{*}-E X_{n-i: n}^{*}\right)$ is non-decreasing in $n$.

As a second example consider the case where $F^{*}$ denotes the exponential distribution function. Then condition (1.3) is satisfied if the distribution $F$ has increasing failure rate

$$
q(x)=F^{\prime}(x) /(1-F(x))
$$

(cf. [1] or [5]). We have (cf. similar results in [1]): If $F$ has increasing failure rate, then $(n-i)\left(E X_{i+1: n}-E X_{i: n}\right)$ is non-increasing in $i$ and non-decreasing in $n$, whereas $\left(E X_{n-i+1: n}-E X_{n-i: n}\right)$ is non-increasing in $n$.

For other cases where relations (1.3) or (1.4) are satisfied and the results of this paper may be applied, the reader is referred to [5].

Acknowledgment. The author is endebted to Professor Richard E. Barlow for a stimulating discussion during which Theorem 2.3 was put into shape.

\section{REFERENCES}

[1] Barlow, R. E. and Proschan, F. (1966). Inequalities for linear combinations of order statistics from restricted families. Ann. Math. Statist. 37 1574-1592. 
[2] HoffFing, W. (1953). On the distribution of the expected values of order statistics. Ann. Math. Statist. 24 93-100.

[3] Karlin, S. (1963). Total positivity and convexity preserving transformations. Convexity, Proc. Symp. Pure Math. 7 329-347. Amer. Math. Soc., Providence.

[4] MolenaAr, W. and van Zwet, W. R. (1966). On mixtures of distributions. Ann. Math. Statist. 37 281-283.

[5] vaN ZWET, W. R. (1964). Convex transformations of random variables. Mathematical Centre Tract 7. Mathematisch Centrum, Amsterdam. 